\title{
国内における抗血清リスト
}

\section{List af Reagents for Animal Blood Group Research}

A. 血液型抗血清 (Blood G roup $R$ eagen $\mathrm{t} s$ )

List of Reagents for Cattle

\begin{tabular}{|c|c|c|c|c|c|}
\hline $\begin{array}{l}\text { Abbreviation } \\
\text { of } \\
\text { laboratory }\end{array}$ & System & An $t$ is e rum & Source & $\mathrm{Nature}$ & Note \\
\hline \multirow[t]{22}{*}{ N I A I } & A & $A_{1}$ & is o i mmune & lys. & \\
\hline & & $\mathrm{A}_{2}$ & is o i mmune & lys. & ava i lable \\
\hline & & $Z^{\prime}$ & is o immune & lys. & available \\
\hline & B & B & i so immune & lys. & avai lable \\
\hline & & $\mathrm{G}_{2}$ & is o immune & lys. & available \\
\hline & & $\mathrm{I}_{1}$ & is o immune & lys. & \\
\hline & & $\mathrm{I}_{2}$ & is o immune & lys. & available \\
\hline & & $\mathrm{K}$ & i s o immune & lys. & \\
\hline & & $\mathrm{O}_{1}$ & i s o i mmune & lys. & \\
\hline & & $\mathrm{T}_{2}$ & is o immune & lys. & \\
\hline & & $\mathrm{Y}_{2}$ & is o i mmune & lys. & ava il able \\
\hline & & $\mathrm{B}^{\prime}$ & i s o i mmune & lys. & \\
\hline & & $\mathrm{D}^{\prime}$ & is o immu ne & lys. & available \\
\hline & & $\mathrm{E}_{2}^{\prime}$ & i s o i mmune & lys. & \\
\hline & & $\mathrm{I}^{\prime}$ & is o immune & lys. & avai lable \\
\hline & & $\mathrm{J}^{\prime}$ & i so immune & lys. & ava i lable \\
\hline & & $\mathrm{K}^{\prime}$ & is o immune & lys. & \\
\hline & & $\mathrm{O}^{\prime}$ & is o immune & lys. & \\
\hline & & $\mathrm{Y}^{\prime}$ & i s o i mmune & lys. & \\
\hline & $\mathrm{C}$ & $\mathrm{C}_{2}$ & i so immune & lys. & ava i lable \\
\hline & & $\mathrm{W}_{1}$ & i s o i mmune & lys. & \\
\hline & & $\mathrm{X}_{1}$ & i s o i mmune & lys. & ava i lable \\
\hline
\end{tabular}




\begin{tabular}{|c|c|c|c|c|c|}
\hline $\begin{array}{c}\text { Abbreviation } \\
\text { of } \\
\text { laboratory }\end{array}$ & Syst em & An $t$ is erum & Source & Nature & Note \\
\hline \multirow[t]{13}{*}{ N IAI } & $\mathrm{C}$ & $\mathrm{R}$ & i s o immune & lys. & \\
\hline & \multirow[t]{3}{*}{ F } & $F_{1}$ & $\begin{array}{l}\text { het eroimmune } \\
(\mathrm{rab} \text { i t })\end{array}$ & lys. & \\
\hline & & $\mathbf{F}_{2}$ & $\begin{array}{l}\text { het e ro immune } \\
(\text { goat })\end{array}$ & lys. & avai lable \\
\hline & & $\mathrm{V}$ & i s o immune & lys. & available \\
\hline & $\mathrm{J}$ & $\mathrm{J}$ & $\begin{array}{l}\text { catt le norma l } \\
\text { serum }\end{array}$ & lys. & avai lable \\
\hline & $\mathrm{L}$ & $\mathrm{L}$ & i s o immu ne & lys. & ava ilable \\
\hline & \multirow[t]{5}{*}{$\mathrm{S}$} & $\mathrm{S}$ & i s o i mmu ne & lys. & \\
\hline & & $\mathrm{H}^{\prime}$ & i soimmune & lys. & \\
\hline & & $\mathrm{U}_{1}$ & i so i mmu ne & lys. & \\
\hline & & $\mathrm{U}_{2}$ & is o i mmu ne & lys. & available \\
\hline & & $\mathrm{U}^{\prime}$ & i so i mmu ne & lys. & a va i lable \\
\hline & $Z$ & Z & i so i mmu ne & lys. & available \\
\hline & $\mathrm{R}^{\prime}$ & $\mathrm{R}^{\prime}$ & i s o i mmune & lys. & ava i lable \\
\hline \multirow[t]{11}{*}{ TUA } & \multirow[t]{2}{*}{ A } & $\mathbf{A}_{2}$ & i so i mmune & lys. & \\
\hline & & $Z^{\prime}$ & i so immu ne & lys. & \\
\hline & \multirow[t]{5}{*}{ B } & $\mathrm{O}_{1}$ & i so immune & lys. & \\
\hline & & $\mathrm{Y}_{2}$ & i so i mmu ne & lys. & \\
\hline & & $\mathrm{D}^{\prime}$ & is o i mmune & $1 \mathrm{ys}$. & \\
\hline & & $\mathrm{E}_{2}^{\prime}$ & i s o immune & lys. & \\
\hline & & $\mathrm{K}^{\prime}$ & is o immune & lys. & \\
\hline & $\mathrm{C}$ & $\mathrm{P}$ & i s o i mmune & lys. & \\
\hline & $\mathrm{F}$ & $\mathrm{V}$ & i s o i mmu ne & lys. & \\
\hline & $\mathrm{J}$ & $\mathrm{J}$ & $\begin{array}{l}\text { cattle normal } \\
\text { serum }\end{array}$ & lys. & \\
\hline & $\mathrm{L}$ & $\mathrm{L}$ & i s o i mmune & lys. & \\
\hline
\end{tabular}


List of Reagents for Water Buffalo

\begin{tabular}{|c|c|c|c|c|c|}
\hline $\begin{array}{c}\text { Abbreviation } \\
\text { of } \\
\text { laboratory }\end{array}$ & Sys tem & An $t$ iserum & Source & Na tụre & Note \\
\hline \multirow[t]{9}{*}{ TUA } & & $\mathrm{Wh}_{1}$ & $\begin{array}{l}\text { het e r o immune } \\
(\text { goa t })\end{array}$ & lys. & \\
\hline & & $\mathrm{Wh}_{2}$ & $\begin{array}{l}\text { he teroimmune } \\
(\text { rabbit) }\end{array}$ & lys. & \\
\hline & & $\mathrm{Wh}_{3}$ & $\begin{array}{l}\text { he teroimmune } \\
(\text { rabbi t })\end{array}$ & lys. & \\
\hline & & $\mathrm{Wh}_{3}$. & $\begin{array}{l}\text { water buffalo } \\
\text { normal serum }\end{array}$ & lys. & \\
\hline & & $\mathrm{Wh}_{4}$ & $\begin{array}{l}\text { het e roimmune } \\
(\text { rabbi t })\end{array}$ & lys. & \\
\hline & & $\mathrm{Wh}_{5}$ & i so immune & lys. & \\
\hline & & $A x_{1}$ & i so immune & lys. & \\
\hline & $\mathbf{J}$ & $\mathrm{Wb} J$ & $\begin{array}{l}\text { watet buffalo } \\
\text { normal serum }\end{array}$ & lys. & \\
\hline & & $\mathbf{J}$ & $\begin{array}{l}\text { cat t le norma } 1 \\
\text { se rum }\end{array}$ & lys. & \\
\hline
\end{tabular}

List of Reagents for Horse

\begin{tabular}{|c|c|c|c|c|c|}
\hline $\begin{array}{c}\text { Abbreviation } \\
\text { of } \\
\text { laboratory }\end{array}$ & Sys tem & Ant is e rum & Source & Nature & Note \\
\hline \multirow[t]{14}{*}{ LRC } & A & $\mathrm{Aa}$ & i s o immune & lys. agg. & \\
\hline & & & $\begin{array}{l}\text { het e ro immune } \\
(\text { rabbi t })\end{array}$ & & \\
\hline & & $\mathrm{Ab}$ & i s o immune & lys. & \\
\hline & & Ac & i so immune & $1 \mathrm{ys}$. & \\
\hline & $\mathrm{C}$ & $\mathrm{Ca}$ & i so immune & agg. lys. & \\
\hline & & & $\begin{array}{l}\text { he e e roimmune } \\
(\mathrm{rabb} \text { i t })\end{array}$ & & \\
\hline & D & $\mathrm{Da}$ & is o immune & agg. & \\
\hline & & $\mathrm{Db}$ & is o immu ne & ag g: & \\
\hline & & Dc & i so immu ne & agg. & \\
\hline & & Df & iso i mmune & agg. & \\
\hline & & $\mathrm{Dg}$ & is o i mmu ne & agg. & \\
\hline & & $\mathrm{Dk}$ & is o immune & agg. & \\
\hline & & Dl & i s o immune & agg. & \\
\hline & $P$ & $\mathrm{~Pa}$ & is o immune & ly s. & \\
\hline
\end{tabular}




\begin{tabular}{|c|c|c|c|c|c|}
\hline $\begin{array}{c}\text { Abbreviation } \\
\text { of } \\
\text { laboratory }\end{array}$ & Syst em & Ant i serum & Source & Nature & Note \\
\hline \multirow[t]{3}{*}{ LRC } & $\mathbf{P}$ & $\mathrm{U}_{6}$ & i s o i mmune & lys. & \\
\hline & Q & Q a & i s o i mmune & lys. & \\
\hline & $\mathrm{U}$ & $\mathrm{Ua}$ & i s o immu ne & lys. & \\
\hline
\end{tabular}

List of Reagents for Sheep

\begin{tabular}{|c|c|c|c|c|c|}
\hline $\begin{array}{c}\text { Abbreviation } \\
\text { of } \\
\text { laboratory }\end{array}$ & Sys tem & Antiserum & Source & Nature & Note \\
\hline \multirow[t]{9}{*}{ N IA I } & $\mathrm{R}-\mathrm{O}$ & $\mathrm{R}^{*}$ & i s o i mmune & lys. & \\
\hline & & $\mathrm{O}$ & i so immune & lys. & \\
\hline & A & Aa & i so immune & lys. & ava i lable \\
\hline & & $\mathrm{Ab}$ & i s o immune & lys. & \\
\hline & $\mathrm{C}$ & $\mathrm{Ca}$ & i s o i mmune & lys. & available \\
\hline & & $\mathrm{Cb}$ & is o i mmune & lys. & \\
\hline & $\mathbf{M}$ & $\mathrm{Ma}$ & i s o i mmune & lys. & available \\
\hline & & $\mathrm{Mb}$ & i so i mmune & lys. & \\
\hline & & $\mathrm{Mc}_{\mathrm{c}}$ & i s o i mmune & ly s. & \\
\hline
\end{tabular}

* These standard reagents were supplied from Dr. E. M. Tucker.

List of Roagents for Goat

\begin{tabular}{|c|c|c|c|c|c|}
\hline $\begin{array}{l}\text { Abbreviation } \\
\text { of } \\
\text { labora tory }\end{array}$ & System & Antiserum & Source & $\mathrm{Nature}$ & Note \\
\hline \multirow[t]{5}{*}{ TUA } & & $\mathrm{Gh}_{1}$ & i s o i mmu ne & lys. & ava i lable \\
\hline & & $\mathrm{Gh}_{2}$ & i so immune & lys. & ava i lable \\
\hline & & $\mathrm{Gh}_{3}$ & i s o immu ne & lys. & ava ilable \\
\hline & & $\mathrm{Gh}_{4}$ & i s o immu ne & lys. & ava ilable \\
\hline & & $\mathrm{Gh}_{5}$ & is o immune & lys. & available \\
\hline
\end{tabular}

List of Reagents for Swine

\begin{tabular}{cccccc}
\hline \hline $\begin{array}{c}\text { Abbreviation } \\
\text { of } \\
\text { laboratory }\end{array}$ & System & Ant iserum & Source & Nature & Note \\
\hline NIAI & A & A & $\begin{array}{l}\text { swine and cattle } \\
\text { normal sera } \\
\text { isoimmune }\end{array}$ & $\begin{array}{l}\text { lys.\& } \\
\text { agg. } \\
\text { lys. }\end{array}$ & available \\
\hline
\end{tabular}




\begin{tabular}{|c|c|c|c|c|c|}
\hline $\begin{array}{c}\text { Abbreviation } \\
\text { of } \\
\text { laboratory }\end{array}$ & Sys tem & Ant ise rum & Source & Nature & Note \\
\hline \multirow[t]{21}{*}{ NIAI } & \multirow[t]{7}{*}{$\mathrm{E}$} & $\mathrm{Ea}$ & $\begin{array}{l}\text { i solimmune } \\
\text { het eróimmune } \\
\text { (rabbit) }\end{array}$ & agg. & available \\
\hline & & $\mathrm{Eb}$ & $\begin{array}{l}\text { is o immu ne } \\
\text { heteroimmune } \\
\text { (rabbit) }\end{array}$ & agg. & available \\
\hline & & Ed & i s o i mmune & agg. & available \\
\hline & & $\mathrm{Ee}$ & i s o immune & agg. & available \\
\hline & & Ef & is o immune & agg. & \\
\hline & & $\mathrm{Eg}$ & i s o i mmune & agg. & ava ilable \\
\hline & & $\mathrm{Em}$ & is o immune & agg. & \\
\hline & \multirow[t]{2}{*}{$\mathrm{F}$} & $\mathrm{Fa}$ & is o immune * & agg. & \\
\hline & & $\mathrm{Fb}$ & i s o immune & agg. & ava i lable \\
\hline & $\mathrm{G}$ & $\mathrm{Ga}$ & is o i mmune & coombs & ava ilable \\
\hline & \multirow[t]{2}{*}{$\mathrm{H}$} & $\mathrm{Ha}$ & is o immune & l ys. & \\
\hline & & $\mathrm{Hb}$ & is o i mmune & lys. & \\
\hline & \multirow[t]{2}{*}{$\mathrm{K}$} & $\mathrm{Ka}$ & $\begin{array}{l}\text { i so immune } \\
\text { heteroimmune } \\
\text { (rabbit) }\end{array}$ & lys. & ava i lable \\
\hline & & $\mathrm{Kb}$ & $\begin{array}{l}\text { is so immúne } \\
\text { he e eroimmune } \\
\text { (rabbit) }\end{array}$ & lys. & ava i lable \\
\hline & \multirow[t]{3}{*}{$\mathbf{L}$} & $\mathrm{La}$ & is o immune & a gg. & \\
\hline & & Lh & i so i mmune & dext. & \\
\hline & & $\mathrm{Lk}$ & is o immune & a gg. & \\
\hline & $\mathrm{O}$ & $\mathrm{Oa}$ & is o immune * & agg. & \\
\hline & \multirow[t]{2}{*}{$P S A-I * *$} & I a & is o i mmune & precipit in & available \\
\hline & & $\mathrm{Ib}$ & is o i mmune & precipitin & available \\
\hline & $\mathrm{PSA}-I^{* *}$ & II $\mathbf{a}$ & is o immune & precipit in & avai lable \\
\hline \multirow[t]{7}{*}{$\mathrm{T}$} & \multirow[t]{2}{*}{ A } & A & $\begin{array}{l}\text { swine normal } \\
\text { serum }\end{array}$ & $\begin{array}{l}\text { lys. \& } \\
\text { agg. }\end{array}$ & available \\
\hline & & $\mathrm{O}$ & i s o immune & agg. & \\
\hline & \multirow[t]{2}{*}{ B } & $\mathrm{Ba}$ & is o immune & agg. & available \\
\hline & & $\mathrm{Bb}$ & $\begin{array}{l}\text { he teroimmune } \\
(\text { rabbit })\end{array}$ & agg. & \\
\hline & \multirow[t]{3}{*}{$\mathrm{E}$} & $\mathrm{Ea}$ & i s o immun e & agg. & ava ilable \\
\hline & & $\mathrm{Eb}$ & i s o immune & agg. & avai lable \\
\hline & & $\mathrm{Ed}$ & i s o immune & agg. & available \\
\hline
\end{tabular}




\begin{tabular}{|c|c|c|c|c|c|}
\hline $\begin{array}{c}\text { Abbreviation } \\
\text { of } \\
\text { laboratory }\end{array}$ & System & Ant is erum & Source & Nature & Note \\
\hline \multirow[t]{17}{*}{$T$} & $\mathrm{E}$ & $\mathrm{E} \mathrm{e}$ & i s o immune & agg. & available \\
\hline & & $E_{f}$ & i s o i mmu ne & agg. & ava ilable \\
\hline & & $\mathrm{Eg}$ & i s o i mmu ne & agg. & a va il able \\
\hline & & $\mathrm{Eh}$ & i s o i mmune & agg. & \\
\hline & F & $\mathrm{F} \mathrm{a}$ & i so i mmune & agg. & available \\
\hline & & $\mathrm{F} \mathrm{b}$ & i s o i mmu ne & agg. & available \\
\hline & G & $\mathrm{Ga}$ & i s o immu ne & agg. & \\
\hline & & $\mathrm{Gb}$ & i s o i mmune & agg. & ava ilable \\
\hline & $\mathbf{H}$ & $\mathrm{Ha}$ & is o i mmu ne & lys. & \\
\hline & & $\mathrm{Hb}$ & i s o immu ne & lys. & available \\
\hline & $\mathrm{K}$ & $\mathrm{Ka}$ & $\begin{array}{l}\text { i so immune } \\
\text { het ero immune } \\
\text { (rabbi t) }\end{array}$ & lys. & ava ilable \\
\hline & & $\mathrm{Kb}$ & $\begin{array}{l}\text { is so immúne } \\
\text { heteroimmu ne } \\
\text { (rabbit) }\end{array}$ & lys. & ava i lable \\
\hline & & $\mathrm{Ke}$ & is o immune & agg. & \\
\hline & $\mathrm{L}$ & $\mathrm{Lg}$ & i s o i mmune & agg. & available \\
\hline & & $\mathrm{Lh}$ & i so immune & c oom bs & a va i lable \\
\hline & & $\mathrm{Lk}$ & i s o immu ne & $\begin{array}{l}\mathrm{agg} \cdot \& \\
\mathrm{c} \mathrm{oomb} \mathrm{s}\end{array}$ & available \\
\hline & & L l & i s o i mmune & agg. & \\
\hline
\end{tabular}

* These antisera were i solated from the sera of $\mathrm{s}$ ows which gave birth to piglets affected hemplytic disease.

**Porcine serum allotype.

List of Reagents for Chickens

\begin{tabular}{|c|c|c|c|c|c|}
\hline $\begin{array}{c}\text { Abbreviation } \\
\text { of } \\
\text { laboratory }\end{array}$ & Sy st em & Ant is er um & Sou rce & $\mathrm{Nat}$ ure & Note \\
\hline \multirow[t]{4}{*}{$\mathrm{H}$} & A & F & i so immune & agg. & \\
\hline & & $\mathrm{H}$ & i so immune & a gg. & \\
\hline & & $\mathrm{R}$ & i s o immune & agg. & avai lable \\
\hline & & $\mathrm{P}$ & is o immune & a gg. & \\
\hline
\end{tabular}




\begin{tabular}{|c|c|c|c|c|c|}
\hline $\begin{array}{c}\text { Abbreviation } \\
\text { of } \\
\text { laboratory }\end{array}$ & System & Ant is erum & Source & Nature & Note \\
\hline \multirow[t]{26}{*}{$\mathrm{H}$} & A & $Z$ & i s o immune & agg. & ava i lable \\
\hline & & $C^{\prime}$ & is o i mmune & agg. & ava i lable \\
\hline & & $\mathrm{D}^{\prime}$ & i s o immune & ag g. & \\
\hline & & $\mathrm{E}^{\prime}$ & i s o i mmu ne & agg. & a va i lable \\
\hline & & $\mathrm{H}^{\prime}$ & i s o immune & agg. & \\
\hline & B & A & i s o immune & agg. & \\
\hline & & B & i s o i mmune & agg. & \\
\hline & & $\mathrm{C}$ & i s o immu ne & agg. & \\
\hline & & D & is o immune & agg. & \\
\hline & & $\mathrm{E}$ & is o immune & agg. & ava i lable \\
\hline & & G & is o i mmune & agg. & \\
\hline & & I & is o immune & agg. & available \\
\hline & & $\mathrm{K}$ & i s o immune & agg. & available \\
\hline & & $\mathrm{L}$ & is o i mmune & agg. & ava il able \\
\hline & & M & i s o immune & agg. & a vailable \\
\hline & & $\mathrm{N}$ & i s o immune & agg. & a va i lable \\
\hline & & $\mathrm{O}$ & i s o i mmu ne & agg. & \\
\hline & & $\mathrm{T}$ & is o i mmune & agg. & \\
\hline & & $\mathrm{A}^{\prime}$ & i s o i mmune & agg. & avai lable \\
\hline & & $\mathrm{J}^{\prime}$ & i s o i mmu ne & agg. & \\
\hline & & $\mathrm{K}^{\prime}$ & i s o immune & agg. & a $\nabla a$ i $l a b l e$ \\
\hline & & $\mathrm{L}^{\prime}$ & i s o i mmune & agg. & \\
\hline & $\mathrm{D}$ & Q & i s o immune & agg. & \\
\hline & & $\mathrm{S}$ & is o immune & agg. & available \\
\hline & $\mathrm{E}$ & W & i s o i mmu ne & ag g. & \\
\hline & & Y & i s o immune & agg. & \\
\hline \multirow[t]{2}{*}{ N IBS } & $\mathrm{Ph}$ & PHA & g arden pea & agg. & available \\
\hline & $\mathrm{Pn}$ & PHA & peanut & agg. & ava i lable \\
\hline
\end{tabular}




\begin{tabular}{|c|c|c|c|c|c|}
\hline $\begin{array}{c}\text { Abbreviation } \\
\text { of } \\
\text { laboratory }\end{array}$ & Sys tem & Ant is erum & Source & Nature & Note \\
\hline \multirow[t]{9}{*}{ NI BS } & $\mathrm{Tg}$ & PHA & t ul ip & agg. & ava i lable \\
\hline & $\mathrm{St}_{1}$ & PHA & potato & agg. & ava i lable \\
\hline & $\mathrm{St}_{2}$ & PHA & potato & a gg. & a va i lable \\
\hline & $\mathrm{S} \mathrm{i}$ & PHA & sesame & agg. & ava i lable \\
\hline & $\mathrm{Ln}$ & PHA & $\begin{array}{l}\text { Lepsta nuda } \\
\text { (Murasakishime ji) }\end{array}$ & agg. & available \\
\hline & A t & PHA & Aliium tuberosum & agg. & available \\
\hline & $\mathrm{Pw}$ & PHA & p ok ewe ed & agg. & available \\
\hline & Gs & PHA & soybean & a gg. & avai lable \\
\hline & $\mathrm{Va}$ & PHA & $\begin{array}{l}\text { Iscum al lbmin } \\
\text { (Yadorig i) }\end{array}$ & agg. & a va il able \\
\hline
\end{tabular}

List of Reagents for Japanese Quail

\begin{tabular}{|c|c|c|c|c|c|}
\hline $\begin{array}{c}\text { Abbreviation } \\
\text { of } \\
\text { laborat or y }\end{array}$ & Sys tem & An $t$ is e rum & Source & Nature & Note \\
\hline \multirow[t]{4}{*}{ N I BS } & Pn & PHA & peanut & ag g. & available \\
\hline & $\mathrm{Sb}$ & PHA & s pybean & ag $g$. & available \\
\hline & Ns & PHA & $\begin{array}{l}\text { Naematolama } \\
\text { ietur } b^{-} \\
\text {(Kurtitum } \\
\text { (Kuritake) }\end{array}$ & ag $g$. & a vailable \\
\hline & $\mathrm{Ht}$ & PHA & $\begin{array}{l}\text { H lianthus tuberosits } \\
\text { (Ki ku imo) }\end{array}$ & agg. & avai lable \\
\hline
\end{tabular}

List of Reagents for Pigeons

\begin{tabular}{|c|c|c|c|c|c|}
\hline $\begin{array}{l}\text { Abbreviation } \\
\text { of } \\
\text { laboratory }\end{array}$ & Syst em & Ant is erum & Source & $\mathrm{Nature}$ & Note \\
\hline \multirow[t]{5}{*}{ NIBS } & $\mathrm{Pc}$ & PHA & yarden pea & a gg. & available \\
\hline & Dt & PHA & $\begin{array}{l}\text { () loscorea batatas } \\
\text { Nagaimo) }\end{array}$ & agg. & available \\
\hline & $\mathrm{Cc}$ & PHA & (Hoteishime j i) & agg. & available \\
\hline & $\mathrm{Am}$ & PHA & Amaryllis & agg. & avai lable \\
\hline & $\mathrm{Cr}$ & PHA & Crocus & agg. & a vai lable \\
\hline $\begin{aligned}<\text { 略記号> } & \text { agg } \\
& \text { lys } \\
& \operatorname{coc}\end{aligned}$ & $\begin{array}{r}: \text { ag } \\
: \text { her } \\
\text { hbs }: \text { in }\end{array}$ & $\begin{array}{l}\text { t inin } \\
\text { lysin } \\
\text { mplete aggl }\end{array}$ & $\begin{array}{l}\text { dext. } \\
\text { a vai lable } \\
\text { utinin } \text { N. I. }\end{array}$ & $\begin{array}{l}\text { dextran } \\
: \text { for stuc } \\
\text { not ider }\end{array}$ & $\begin{array}{l}\text { agglutin in } \\
\text { ly } \\
\text { t if ied }\end{array}$ \\
\hline
\end{tabular}


B. 一般抗血清 (Other an $\mathrm{t}$ ise ra )

\begin{tabular}{|c|c|c|c|c|}
\hline $\begin{array}{l}\text { Abbreviation } \\
\text { of } \\
\text { l abor atory }\end{array}$ & Specif ic ity & Source & Nature & No te \\
\hline \multirow[t]{7}{*}{ NIA I } & Ant $\mathrm{i}$-rabbi t $\mathrm{C}_{3}$ & het eroimmune & Precipitin & avai lable \\
\hline & Ant i-rabbit $\mathrm{C}_{6}$ & is o immune & Precipitin & a vai lable \\
\hline & Anti-cattle $\mathrm{C}_{3}$ & heteroimmune & Precipitin & avai lable \\
\hline & Ant $i-c a t t l e$ transferrin & heteroimmune & Precipitin & ava i lable \\
\hline & Anti-cattle $\alpha$-feto protein & heteroimmune & Precipitin & available \\
\hline & Ant $i-c a t t l e$ IgG & he teroimmune & Precipit in & available \\
\hline & Anti-chicken $\mathrm{C}_{3}$ & het er oimmune & Precipitin & available \\
\hline
\end{tabular}

抗血清の所有研究機関とその略号

$\mathrm{H}$ : 広島大学生物生産学部家畜育種学教室 (岡田育穂)

于7 20 福山市緑町 2-17

電 $(0849) 24-6211$

L R C : 競走馬理化学研究所血液型部（茂木一重）

干158 東京都世田谷区用賀 4-37-6

電(03) $429-4191$

N I A I ：農林水産省畜産試験場育種部（阿部恒夫）

干 305 茨城県稲敷郡茎崎町池の台 2 農林研究団地内局 私書箱 5 号

電 (02975) 6-8620

\section{N I BS：日本生物科学研究所附属実験動物研究所（水谷 誠)}

干 409-16 山梨県北巨摩郡小㴊沢町上笹尾 3331 -114 電 (055136) 2333

$\mathrm{T}$ : 北海道立滝川畜産試験場養豚科（山田 渥）

干 073 北海道滝川市東滝川 735

電 $(01252) 2-2171$

TUA : 東京農業大学家畜育種学研究室 (天野 卓)

干156 東京都世田谷区桜丘 1-1-1

電 (03) $420=2131$ 